\title{
Cardiovascular risk and physical therapy in primary prevention
}

\author{
Peres Paulo* \\ Universidade Nove de Julho - UNINOVE, São Paulo, Brazil
}

Cardiovascular Artery Disease (CAD) is the leading cause of death among chronic-degenerative diseases in most countries. The treatment, aimed at primary care and prevention, consists of exercises to combat sedentary lifestyle, proper nutrition, stop smoking and weight control $[1,2]$.

The objective of this study is to evaluate the anthropometric and physiological variables, glycemia, cholesterol and triglycerides, quality of life and international classification of functionality in adults and to guide the practice of simple physiotherapeutic exercises through a booklet.

This is a randomized, controlled, blind trial with individuals recruited from the university or extra-university environment. Adults of both sexes, age from 20 and 60 years, and sedentary will be included. Exclusion criteria are subjects with excessive discomfort to the digital puncture, with incapacity to understand the booklet, that prevent the accomplishment of any step of the study, with heart cardiovascular disease proven or in use of any medication and subjects with respiratory, genetic, hematological and oncological diseases. This study was approved by the ethics committee (No. 2962841) and it is registered in the REBEC (U1111-1220-7494).

According to sample size calculation, a total of 341 individuals will be divided into two groups. Weight, height, body mass index (BMI), waist/hip ratio, blood pressure and resting heart rate, and SF-36 questionnaire will be assessed pre and post three months of intervention. Also, blood samples will be collected through digital puncture for evaluation of total cholesterol, triglycerides and glycemia. Subjects will be randomized for either experimental group (171 individuals) or control group (170 individuals) (Figure 1). Subjects in experimental group will receive an orientation booklet for practicing localized exercises and an aerobic activity. The four exercises should be performed in a total time of two minutes as follows: squatting with wall support, standing on the tip of the foot (if necessary with support), flexing of the elbows in the wall slightly away from it (arm flexion), elevation lateral of the arms with load of half kilogram in the first and second month and with 1 kilogram in the third month. Each exercise will have 20 repetitions of 30 seconds each; at the end of the exercises a seven-minute stationary walk will

Copyright: (C2019 Paulo P. This is an open-access article distributed under the terms of the Creative Commons Attribution License, which permits unrestricted use, distribution, and reproduction in any medium, provided the original author and source are credited.

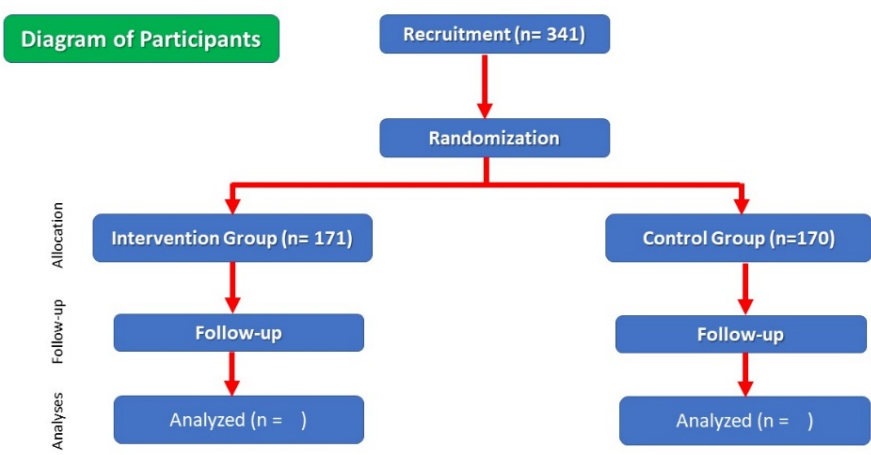

Figure 1. Flow chart of the study

compose the aerobic activity in the first month. In the second month, the same exercises will be performed with 30 repetitions in the same time interval and at the end a 12-minute stationary walk. In the third month, the same exercises will be performed with 40 repetitions and at the end 17 minutes of standing stationary. Control group will receive a folder with guidelines for the regular practice of resistance and aerobic exercises. Both groups will receive a score sheet for the days on which they performed the exercises and the level of fatigue according to the modified Borg table (from 0 to 10). Individuals will be instructed to level at four to five according to the table and perform the exercises with a frequency of three to five times a week.

The primary outcome is (1) the assessment of cardiovascular risk (reduction of total cholesterol levels, triglycerides to normal values or at least $20 \%$ of the initial values), (2) a $10 \%$ loss in body weight, if overweight, and (3) adherence to exercise assessed by completing $\geq$ $70 \%$ of the follow-up form at the end of the third month. The secondary outcome is an improvement in quality of life evaluated by the SF 36 and cardiovascular risk questionnarie.

\section{References}

1. Sociedade Brasileira de Cardiologia (2014) Diretriz Sul-Americana De Cardiovascular Prevenção E Reabilitar Cardiovascular. Arq Bras Cardiol 103: 1-31.

2. Goble AJ, Worcester MUC (1999) Best Practice Guidelines for Cardiac Rehabilitation and Secondary Prevention. Hum Serv Victoria 1-238.

*Correspondence to: Peres Paulo, Universidade Nove de Julho, UNINOVE: Rua Vergueiro 235/249 - CEP 01504-001 - São Paulo - Brazil, E-mail: pauloperes@uni9.pro.br

Key words: cardiovascular risk, anthropometry, exercise, primary prevention, physical therapy

Received: March 20, 2019; Accepted: March 27, 2019; Published: March 29, 2019 